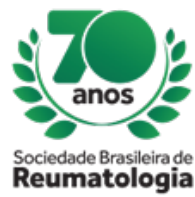

\title{
A RARE PRESENTATION OF GRANULOMATOSIS WITH POLYANGIITIS MIMICKING CERVICAL CANCER
}

Ana Julia Furbino Dias Bicalho (Universidade Federal de Minas Gerais, Belo Horizonte, MG, Brasil),

Deborah Lobato Guimaraes (Universidade Federal de Minas Gerais, Belo Horizonte, MG, Brasil),

Henrique Valladao Pires Gama (Universidade Federal de Minas Gerais, Belo Horizonte, MG, Brasil), Moises Salgado Pedrosa (Universidade Federal de Minas Gerais, Belo Horizonte, MG, Brasil), Daniela de Souza Braga (Universidade Federal de Minas Gerais, Belo Horizonte, MG, Brasil), Clara Rodrigues Alves de Oliveira (Universidade Federal de Minas Gerais, Belo Horizonte, MG, Brasil), Leandro Augusto Tanure (Universidade Federal de Minas Gerais, Belo Horizonte, MG, Brasil), Gustavo Gomes Resende (Universidade Federal de Minas Gerais, Belo Horizonte, MG, Brasil), Gilda Aparecida Ferreira (Universidade Federak de Minas Gerais, Belo Horizonte, MG, Brasil)

\section{BACKGROUND}

Granulomatosis with polyangiitis (GPA) is a small vessels systemic vasculitis. The genital tract is affected in less than $1 \%$ of patients, according to previous studies, of which uterine cervix involvement is exceedingly rare. We describe the case of a patient with genital involvement as a late manifestation of GPA.

\section{CASE REPORT}

A 64-year-old female patient with GPA diagnosis since 2008, of which the past history presented necrotizing scleritis, pulmonary and sinonasal involvement, with nasal saddling and septal perforation. CANCA was positive. She had been treated with intravenous methylprednisolone pulse and cyclophosphamide, once at diagnosis, due to scleritis, and then in 2010 for pulmonary involvement. Since upper and lower airway activity was persistent, rituximab was introduced in 2011 and was used until 2016 when it was interrupted due to long-term disease inactivity, methotrexate kept as maintenance therapy. In 2018, she was under investigating for a persistent brownish, non-bullous and odourless vaginal discharge, associated with vegetative cervical lesion, when began again with cough, bloody nasal discharge, wheezing, dyspnoea, fever and prostration, being hospitalized. CT scan revealed chronic acute pansinusopathy, and signals of paranasal cavities and orbits walls reactive osteitis. She developed a palate perforation in which invasive infections were discarded. A first cervix biopsy resulted inconclusive and was not possible to rule out neoplasia. Pelvis MRI revealed $32 \mathrm{~mm}$ circumferential uterine cervix thickening, with anterior vaginal wall in its upper third extension involvement. A second biopsy revealed granulomatous and necrotizing, suppurative chronic active inflammation, associated with vasculitis in small arteries and veins (Figure 1). In this context, was opted for reintroduction of methylprednisolone pulse therapy and oral cyclophosphamide until she has access to rituximab again.

Figure 1. Cervix biopsy. Hematoxylin and Eosin staining (H\&E). Ulcerated uterine cervix, covered by fibrinopurulent exudate, with stroma exhibiting chronic inflammatory infiltrate and geographic pattern necrosis (A). In detail (red square), a medium caliber artery with tumefied wall, containing histiocytes, fibrointimal thickening and fibrinoid necrosis. In its surroundings, loose granuloma and eosinophils are observed (B).

\section{CONCLUSION}

Involvement of the uterine cervix in GPA has been described, but it is an exceedingly rare manifestation. Presents as a component of disease relapse with vaginal bleeding or discharge, as our case. The most important differential diagnosis is carcinoma of the cervix. Infections producing similar appearances should also be excluded. GPA is a multisystem disease and our case reminds the clinician to stay aware of the various presentations of the disease. 